\title{
Standardized Protocol for Hemodialysis Vascular Access Assessment: The Role of Ultrasound and ColorDoppler
}

\author{
Federico Nalesso $^{a, b}$ Francesco Garzotto ${ }^{a, b} \quad$ Ilaria Petruccic Sara Samoni $^{\mathrm{a}, \mathrm{b}}$ \\ Grazia Maria Virzi $^{\mathrm{a}, b}$ Dario Gregori ${ }^{\mathrm{d}}$ Mario Meola ${ }^{c}$ Claudio Ronco ${ }^{\mathrm{a}, \mathrm{b}}$

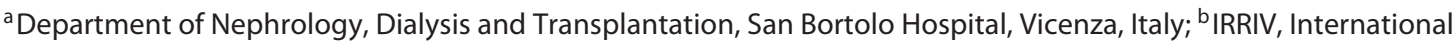 \\ Renal Research Institute Vicenza, Vicenza, Italy; 'Institute of Life Sciences, Sant'Anna School of Advanced Studies, \\ Pisa, Italy; ${ }^{d}$ Biostatistics, Epidemiology and Public Health Unit, Department of Cardiac, Thoracic and Vascular \\ Sciences, University of Padova, Padova, Italy
}

\section{Keywords}

Vascular access · Ultrasound - EchocolorDoppler .

Artero-venous fistula · Graft · Hemodialysis

\begin{abstract}
Introduction: Ultrasound and colorDoppler technique, which is relatively inexpensive, rapid, non-invasive and repeatable is a powerful tool used for early diagnosis of vascular access (VA) complications in hemodialysis patients. To date a standard and widely comprehensible echocolorDoppler (ECD) protocol is not available. Materials and Methods: A simple step-by-step protocol based on anatomical and hemodynamic parameters of VA has been developed during a 3-years VA ECD follow-up. It consists of an ECD study scheme. The algorithm created involves the calculation of brachial artery flow, description of artero-venous and/or graft-vascular anastomosis and efferent vessel and/or graft. Results: The algorithm allows to formulate a medical report that takes into account both anatomic and hemodynamic parameters of the VA. Reduction of complications and the prevention of chronic complications as well as the early detection of acute problems were achieved. Discussion and Conclusion: The
\end{abstract}

(C) 2018 S. Karger AG, Basel

\section{E-Mail karger@karger.com www.karger.com/bpu \\ KARGER}

creation of a step-by-step protocol may simplify the multidisciplinary management of $\mathrm{VA}$, its monitoring and the early diagnosis of its complications.

(c) 2018 S. Karger AG, Basel

\section{Introduction}

The vascular access (VA) is the key element to achieve dialysis efficiency. Blood pump speed, minimum and mean arterial line pressure - both maximum and minimum venous line pressures - were identified as independent determinants for dialysis dose delivery $\mathrm{Kt} / \mathrm{V}$ [1]. All these parameters are strictly correlated with the VA functionality, which in turn demand the clinicians its maintenance and surveillance. The prescription of the dialysis therapy should take into account the maximum achievable blood flow in order to optimize the treatment efficiency and efficacy. In this perspective, VA limitations due to preventable complication must be avoided. VA type and location are the variables to be considered when health-related quality of life is evaluated [2]. Despite all authors agreeing on the importance 
of using strategies to monitor and maintain VA function, there is controversial evidence derived by small studies [3].

The ability to study the VA allows the nephrologist to identify the early dysfunction and establish an effective follow-up [4]. In this way, it is possible to identify the most suitable time to intervention and to correct a malfunction in order to minimize the use of the central venous catheter (CVC) and maintain the patency of the primary VA. The echocolorDoppler (ECD) plays an essential role in the study of VA, providing useful information on its anatomy and hemodynamics, which, integrated with dialysis monitor data, become crucial for the proper follow-up of access $[5,6]$ for the modality of cannulation and for the optimal purification technique according to these elements. Classically, it is known for the utility of the ECD in the study of VA as a non-invasive and repeatable method [7], but it is also attributed to this technique - a significant intra-operator and inter-operator variability that often makes the following-up by different operators difficult [8]. It can determine a not homogeneous and standardized [9] vision overall by the nephrologist and the vascular surgeon. Very often, anatomic alterations identified by the vascular surgeon are not considered hemodynamically relevant for the nephrologist, and vice versa, hemodynamic abnormalities found by the nephrologist are not considered anatomically significant by the vascular surgeon, resulting in a "non-decision" border situation for the management and follow-up in the vascular assess alteration. In these clinical situations, interventional attitudes can be generated on non-hemodynamically significant anatomic defects, especially in stenosis, or attendance attitudes in the case of hemodynamic abnormalities, which may lead to the closing of VA to acute thrombosis $[10,11]$. In that vision, ECD plays an even more significant role in studying the maturation of $\mathrm{VA}$, providing useful information in the identification of early failure and early dysfunction [12], which can be treated surgically in a timely manner, saving the patient from CVC placement with all its complications [10, 11]. The possibility to standardize the ECD analysis determines a clear and reproducible description of vascular alterations that can be treated to maintain the patency and functionality (in terms of adequate blood flow) of the VA.

A unique and standardized ECD use should provide reproducible anatomical and hemodynamic information, both intra- and inter-operator, to perform a more effective analysis in case of acute dysfunction that can also be used in the chronic follow-up. In this point of view, the interdisciplinary approach to the VA plays a synergistic and non-exclusive "hemodynamic and anatomical role," which should always be interpreted not in an "absolute" vision (nephrological or vascular point of view) but in a "clinical and personalized vision" [13].

A VA is often the only option for a provision of lifesaving treatment as dialysis due to the lack of additional options, covering the role of "life-saving VA."

The ECD, with its standardized parameters, becomes an essential and fundamental tool for the study and follow-up of VA by providing the nephrologist and vascular surgeon with information that can be reproduced and analyzed over time.

\section{Methods}

In order to standardize native and prosthetic arteriovenous fistula (AVF) studies, it is mandatory to analyze a well-defined pattern of elements according to a simple, accessible, relatively rapid and repeatable methodology that aims to obtain a standard, clear, complete and reproducible anatomic and hemodynamic description of VA.

For the execution of the present study, according to the proposed protocol, a 7.5 MHz ultrasound (US) linear probe is needed. M-mode, B-mode, Color Doppler and a minimum display facility of the Doppler velocity spectrum for blood flow calculation are mandatory tools.

The proposed protocol allows the study of AVF (native and prosthetic) according to a predetermined step analysis (Table 1) that includes all single elements necessary for the compilation of the report, describing the VA anatomical and hemodynamic characteristics. The protocol provides as first step the identification of the VA at the physical examination in order to describe its position (proximal, proximalized distal, distal) and all pathological elements that can be identified by this procedure. After the physical examination, the VA can be studied step by step in order to describe, in a qualitative and quantitative approach, all anatomical and hemodynamic characteristics.

By a transverse scan in the medial arm region, proceeding from the third proximal to the distal third of the arm, it is necessary to identify the brachial artery. Once the vessel is identified, its diameter, its course and its bifurcation into radial and ulnar arteries should be described. In the middle third of the arm, a measurement of the vessel diameter is carried out using the M-mode to minimize measurement errors and to identify the average diameter in relation to the pulsatility over the time (Fig. 1).

After the measurement of the brachial artery diameter, it is necessary to carry out a longitudinal scan of the artery recording the Doppler velocity spectrum (using an insonation angle $\leq 60^{\circ}$ and a suitable sample volume (Fig. 2) [14]. Once the velocity spectrum is obtained, the calculation of the flow rate can be obtained. The previous measurement of the diameter of the vessel in M-mode minimizes the error, resulting in the scanning of the vessel not on its bigger diameter. This procedure helps in obtaining the best blood flow with the lowest error. The information obtained from this step is essential as allow us to identify an estimated blood flow in the VA and the real resistance in the downstream according to the morphology of Doppler velocity spectrum analysis operated in the brachial artery. 
Table 1. Protocol steps for the analysis of a vascular access

Step-by-step protocol for the assessment of vascular access

1 By a transverse scan:

- identify the brachial artery

Brachial artery

- describe diameter of brachial artery, its course and its bifurcation into the radial and ulnar arteries

- use M-mode* on the brachial artery to identify the average diameter in relation to the pulsatility over the time (Fig. 1)

2 By a longitudinal scan:

- identify the velocity spectrum at the Doppler on brachial artery and provide the calculation of the flow rate*.

The information obtained from this step is essential to identify an estimated blood flow in the vascular access and the real resistance in the downstream according to the morphology of Doppler velocity spectrum* the previous M-mode diameter of brachial artery reduces the error in the calculation of blood flow.

3 Describe the anastomosis between artery (brachial/radial) and vein/graft in terms of 2 diameters ( $\mathrm{mm}$ ) and maximum peak systolic velocity $(\mathrm{cm} / \mathrm{s})$

4 By a longitudinal and traverse scan in B-mode (if required in M-mode):

- Describe the average diameter of the efferent vein/graft, its depth from the skin, its course. According to the minimum diameter of $6 \mathrm{~mm}$ and the maximal depth of $6 \mathrm{~mm}$, identify the best region where proceed with cannulation and verify the blood flow in this region and in the vascular access.

By a longitudinal scan:

- Analyze the wall and the lumen of the access

Describe any alteration in term of structure, dimension, impact on hemodynamics in 2 different planes of scan (aneurysm, pseudo-aneurysm, thrombosis, wall integrity)

- Analyze also the soft tissue peri-access

Describe any alteration in terms of structure, dimension, impact on hemodynamics in 2

different plan of scan (hematoma, peri-access liquid film, edema in the soft tissue)

- Analyze the colorDoppler in order to identify aliasing or black zone in the lumen (stenosis, thrombosis, wall alteration with thrombosis)

5 Describe the anastomosis between the graft and the vein in terms of two diameters ( $\mathrm{mm}$ ) and maximum peak systolic velocity $(\mathrm{cm} / \mathrm{s})$ or between the vein and its confluence in another bigger vessel (generaly a central vein)

Anastomosis at the vein (graft or vein confluence in another vessel)

6 By a transverse scan:

Graft flow

- identify the graft diameter

- use the M-mode* on graft in order to minimize measurement errors in the identification of the average diameter in relation to the pulsatility over the time

- measure the blood flow directly (the graft is not squeezable by the probe as in the case of arteriovenous fistula)

7 By longitudinal and trasverse scan:

Soft tissue and

- identify and describe all alterations in the soft tissue providing their quali-quantitative characteristics in terms accessory of dimension, structure, rrelationship with the access and other anatomical structures vessels

By colorDoppler:

- describe the vascularization of any abnormalities found in the soft tissue and in the perivascular access

By the appropriate scan identify all collateral vessels that can dissipate the blood flow from the effluent vein in order to provide the best follow-up for the access maturation

8 For any alteration found provide:

- B-mode: describe the nature of the alteration and its content (aneurysm, pseudo-aneuriysm, hematoma, fluid collection, liquid film, edema, etc.)

- B-mode in 2 different scan: describe the dimensions (latero-lateral and cranio-caudal diameters, if possible also the antero-posterior diameter)

- ColorDoppler: describe any alteration detectable by this modality (blood flow, blood flow present due to the continuity with the VA or other vessels, etc.) 
Fig. 1. Transverse M-mode scan at the midthird arm. The M-mode measurement of the brachial artery diameter reduces the error and enables the identification of the average diameter in relation to the pulsatility of the vessel.

Fig. 2. Brachial artery flow measurement. Longitudinal scanning of the brachial artery at the middle arm. It is also shown as the Doppler velocity spectrum, using a $57^{\circ}$ insonance angle. The measurement of the diameter of the vessel in M-mode (previously carried out) minimizes the error resulting from a scan non-perfectly along the major diameter of the vessel. This expedient further reduces errors, obtaining a flow closer to the real one.
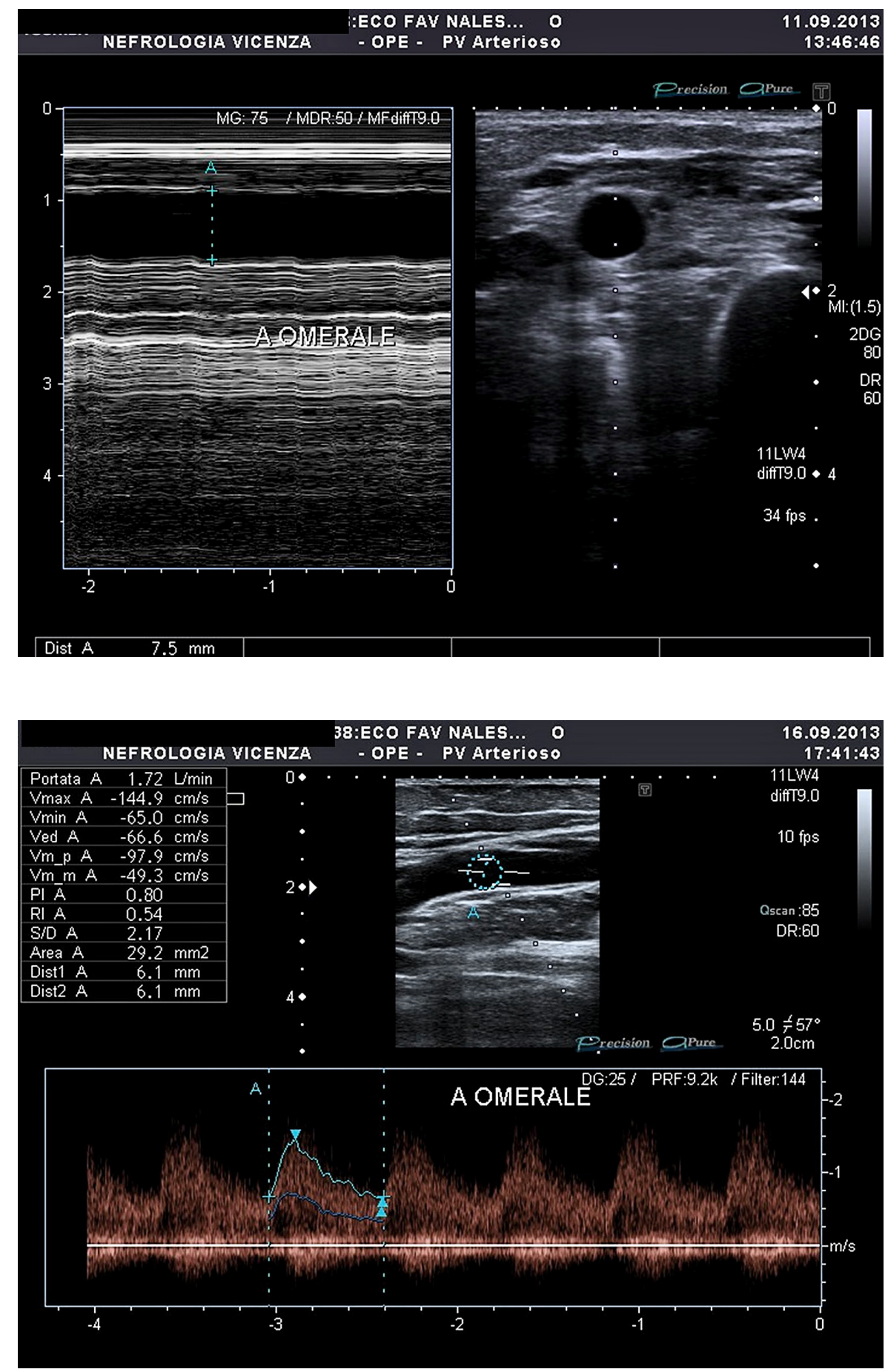

The transversal scanning of the brachial artery (for the proximal access) or the radial artery (for the distal access) in B-mode is performed to identify the anastomosis that can be describe in terms of latero-lateral and cranio-caudal diameters as well as in terms of anatomical structure (latero-lateral, latero-terminal, terminal-terminal). At this anatomical level, it is also necessary to identify any alteration and describe its characteristics (stenosis, dilatation, pseudo-aneurysm). Color Doppler and Doppler veloc- ity should be used in the diagnosis of stenosis identified by both aliasing and systolic peak velocity more than $350 \mathrm{~cm} / \mathrm{s}$ (Fig. 3).

After the anastomosis and the first portion of the efferent vessel study, complete B-mode scan is performed in transverse and longitudinal scan in order to analyze the efferent vein up to its confluence in the central vessel (succlavia vein, axillary vein) or to venous anastomosis in case of prosthesis (Fig. 4). With this step it is possible to describe the vessel wall, its conformation, the presence of stenosis, 
Fig. 3. Stenosis in the peri-anastomotic region. By positioning the sample volume in the presumed stenosis site, the Doppler velocity test detects a systolic peak velocity $(\mathrm{SPV})>450 \mathrm{~cm} / \mathrm{s}$.
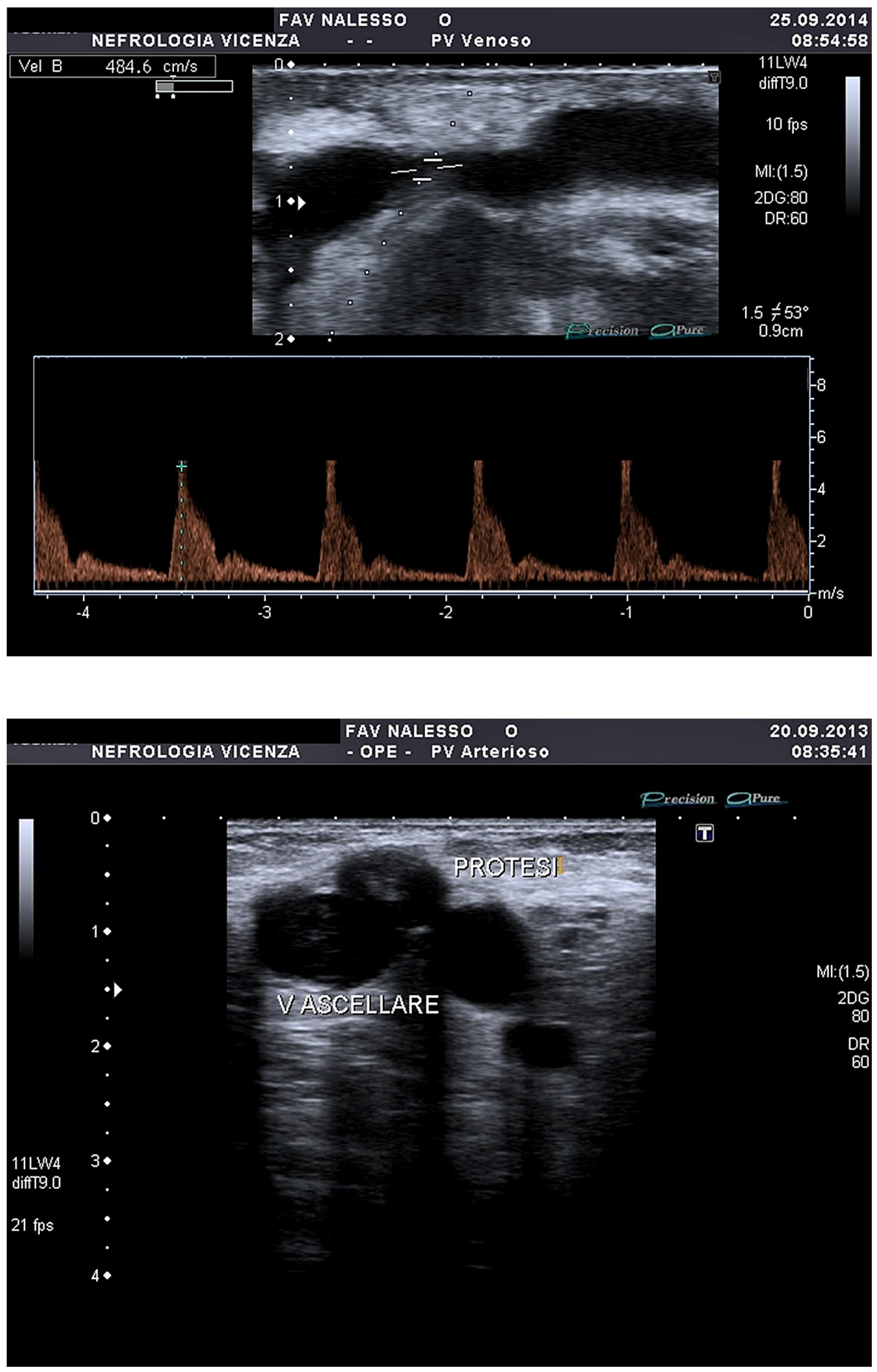

Fig. 4. B-mode scan at the level of anastomosis between graft and axillary vein.

dilations, aneurysms and pseudo-aneurysms whose dimensions have to be measured in the 3 axes. It is also important to describe the presence of collateral vessels, in terms of size, course and dissipation of blood flow from the main vein. After this B-mode study, the colorDoppler assessment is performed in longitudinal scan in order to detect aliasing (it is important to set the correct pulse repetition frequency on the US machine).The Doppler spectrum must have an incidence angle $\leq 60^{\circ}$ at the aliasing zone. In case of stenosis, the sys- tolic peak velocity has to be measured $(\mathrm{m} / \mathrm{s})$ and the demodulation in the Doppler velocity spectrum must be investigated. We highlight that the characteristics of the efferent vein are important to proceed with the cannulation procedure, in the presence of a vessel with a diameter less than $6 \mathrm{~mm}$ and a depth more than $6 \mathrm{~mm}$. The standard cannulation can be problematic and we suggest that the echoguide be used or that a surgeon consulted for an evaluation. The same problem can be present with a graft at more than $6 \mathrm{~mm}$ from the skin. 
Fig. 5. Blood flow of the prosthesis. Longitudinal scan of the prosthesis with Doppler velocity spectrum of the flow using a $60^{\circ}$ angle of insonance and a suitable sample volume.

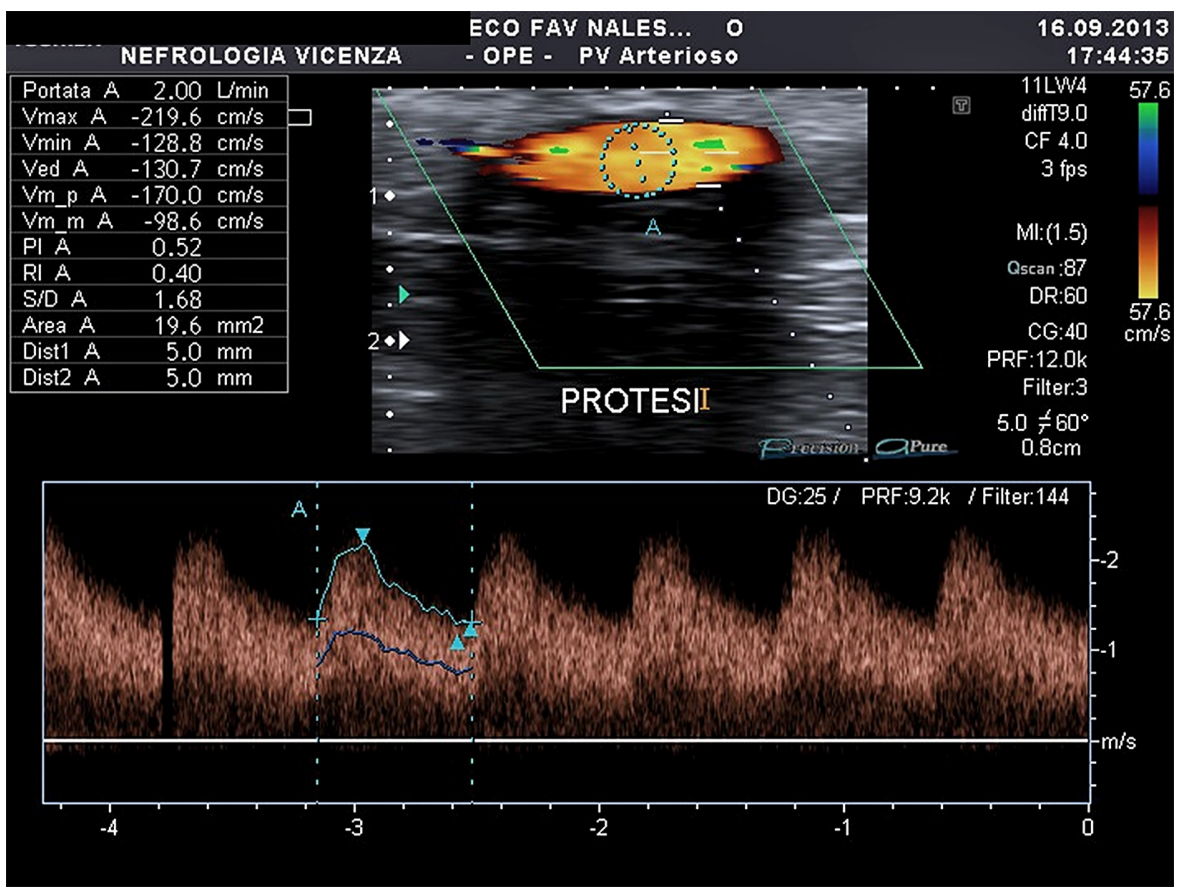

In the case of vascular prosthesis, once the arterial anastomosis has been described and the entire length of the prosthesis has been analyzed, as described above, the venous anastomosis should be described with the same methods used for the arterial anastomosis. In presence of a prosthesis, the blood flow can be calculated directly on the vascular assess, as the diameter of the prosthesis is stable over time (in the native fistula, on the other hand, the vessel diameters change its diameter with the cardiac circle) [15].

The prosthesis flow is calculated using the same procedures described for the artery (Fig. 5). In the case of prosthesis, the diameter is known beforehand. The calculated flow rate in the prosthesis can be compared to that found in the brachial artery.

During the analysis carried out according to the previous points, it is possible to collect information in B-mode and colorDoppler related to the soft tissues surrounding the FAV (hematomas, edema, etc.) and the presence of aneurysms (Fig. 6) and pseudo-aneurysm (Fig. 7), as well as the presence of acute thrombosis with arterial bleeding (Fig. 8). It is necessary to study and describe these alterations with reproducible scans providing information in terms of the size in the 3 axes, wall conformation, tissue reactions, and signal at the color and powerDoppler for the identification of bleeding in the soft tissue. In case of pseudo-aneurysms and aneurysms, the description of the lumen and the identification of endoluminal thrombosis is important for follow-up and treatment.

\section{Results}

Following our VA protocol and processing the obtained qualitative and quantitative parameters, it is possible to formulate a report of the VA that clearly and sim-

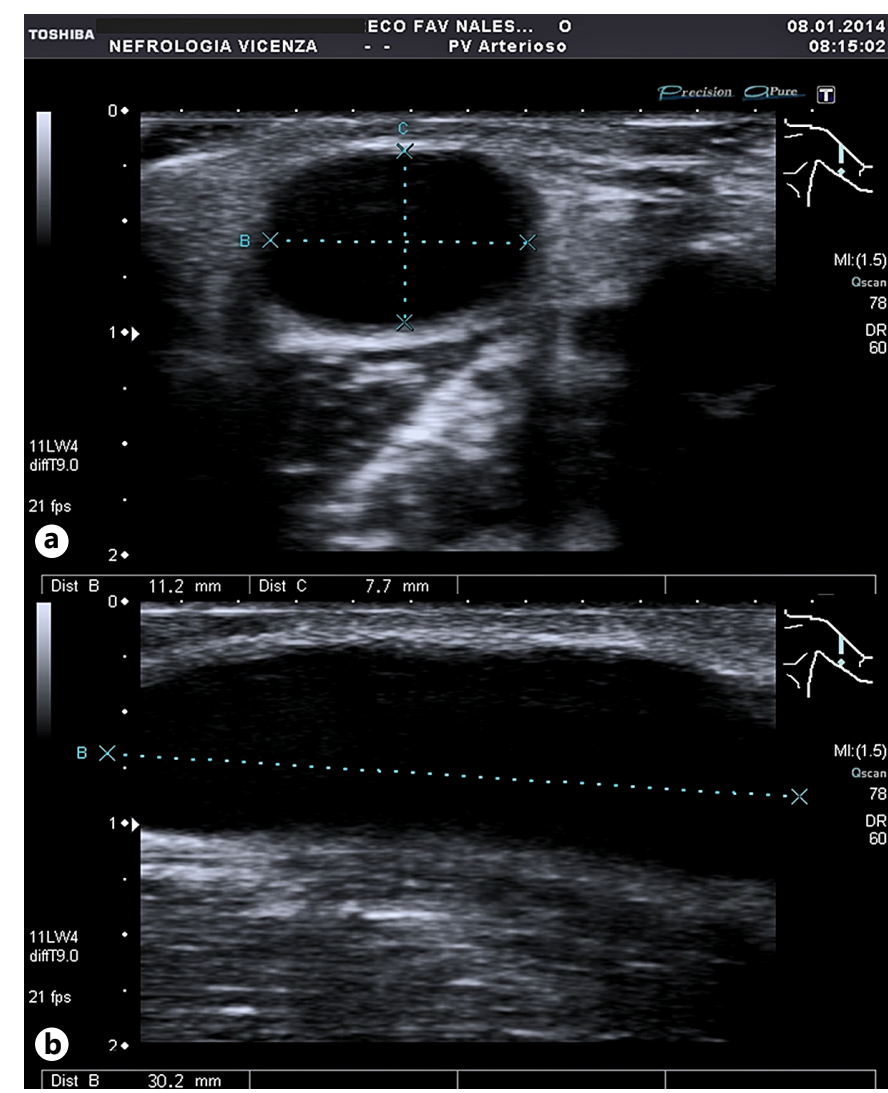

Fig. 6. Transversal (a) and longitudinal (b) scan in B-mode of an aneurysm. For follow-up, it is essential to describe this anatomical alteration with regard to its size in the 3 axes describing the presence of endoluminal thrombosis. 
Fig. 7. Longitudinal scan with a color box of a graft affected by the presence of 2 pseudo-aneurysms. The measurement of their dimensions in the 3 axes leads to their follow-up.

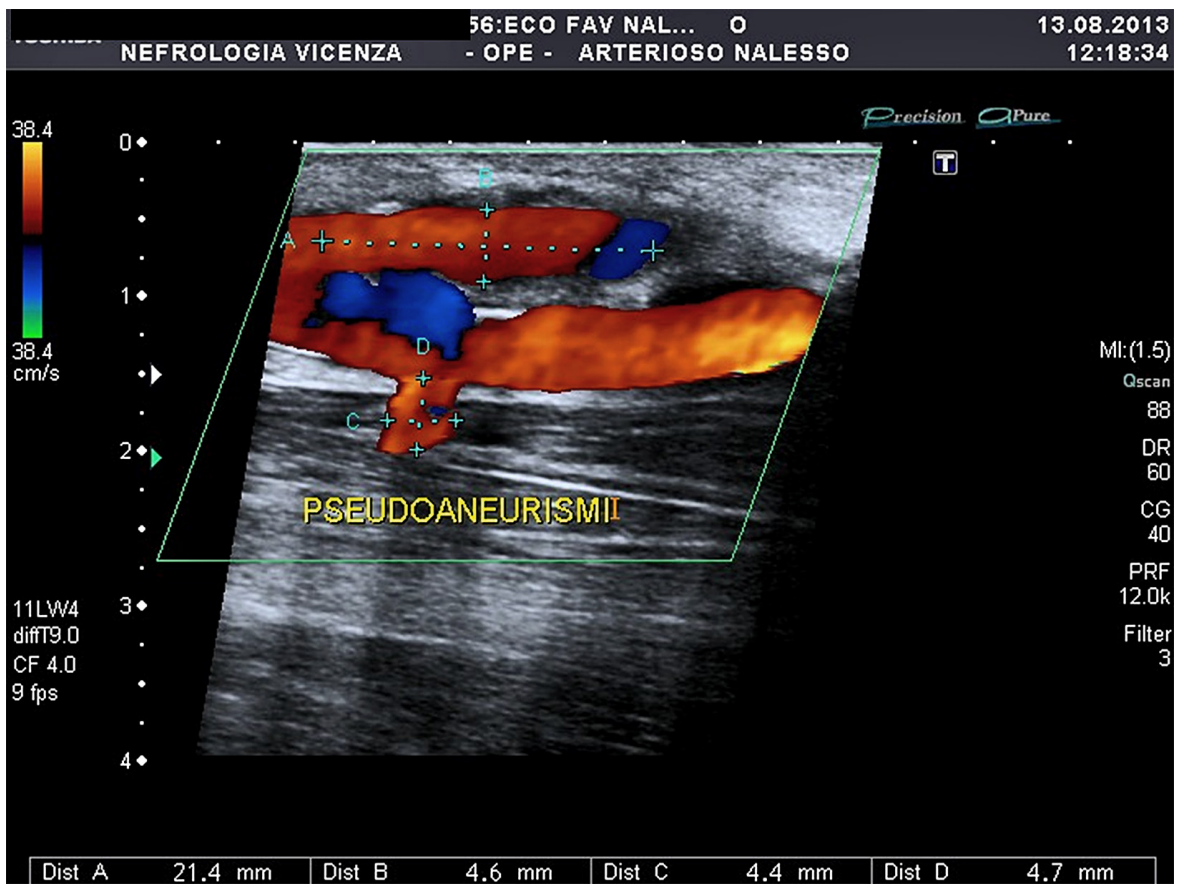

ply describes both anatomical and hemodynamic aspects, providing operational conclusions in a complete and integrated synthesis of its the global function. The protocol steps are shown in Table 1.

The protocol was applied for 3 years in a standard Hemodialysis Unit from January 2015 to November 2017 (Table 2). The total number of patients was 117 on 2015 with 447 ECD provided on 45, 117 on 2016 with 589 ECD on 50 patients and 119 on 2017 with 340 ECD on 35 patients. CVC complications decreased from 158 to 106 over the 3 years ( $-33 \%$ of complications) as the number of CVC placements due to acute or chronic dysfunctions leading to access failure. The reduction of complications is accompanied by an increased number of cannulation provided by the echoguide due to the early detection of evolutive anatomical and hemodynamic problems. In this way, the identification of optimal site of cannulation reduced the number of AVF injuries and their chronic evolutions (aneurysm, pseudo-aneurysm, focal stenosis). The best cannulation for each patient was obtained, thanks to nurses trained to utilize echo-guided procedures in critical situations.

A map of all the available sites for the cannulation results in a tool useful to manage all access with a particular anatomical characteristic. Echoguide procedure can reduce the complications, also allowing frequent changes of the cannulation site in extremely critical situations such as vessel lumen less than $6 \mathrm{~mm}$ and a depth more than
$6 \mathrm{~mm}$ or very short puncture veins. Buttonhole cannulations were successfully done by ECD in very short span of time.

The possibility to follow-up stenosis with a reproducible protocol allowed the early identification of patients with a stenosis evolving in hemodynamical instability and an associated risk of early thrombosis. Six patients were treated by angioplasty at the critical blood flow reduction; only one patient required a new access due to the presence of other anatomical alterations (big aneurysm and pseudo-aneurysms).

The standard introduction of ECD analysis in case of difficult cannulation reduced the aneurysm formation and its enlargement due to the repeated cannulation in the same area. Furthermore, the possibility to use the US before, during and after the cannulation procedure reduces the incidence of pseudo-aneurysm and the wall damage with the formation of hematoma in the soft tissue. In 2015, all nurses of the Nephrology Unit were trained in a theoretical and practical ECD course for the AVF cannulation. In 2016, all nurses and nephrologists of the Unit were trained in a theoretical and practical course to use the ECD in the cannulation, follow-up and assessment of AVF, with particular attention to the blood flow measurement. Introducing this ECD skill in all caregivers of hemodialysis improved the culture of the VA in terms of function, preservation and follow-up. All the complications were 


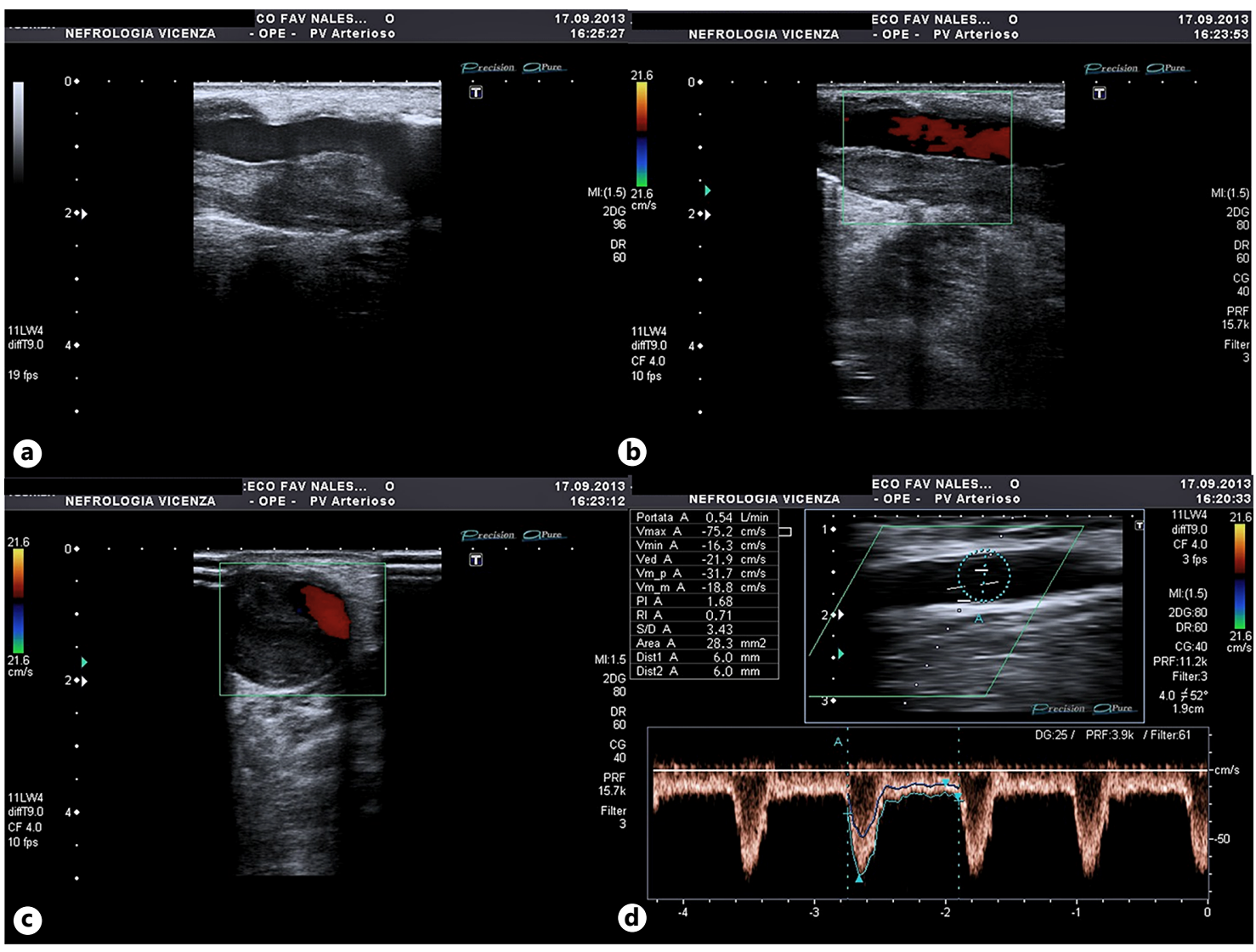

Fig. 8. Longitudinal scan in B-mode of an arteriovenous fistula with endoluminal thrombosis (a). At the color box, the scan in longitudinal (b) and transverse (c) modality confirms the presence of endoluminal thrombosis with minimal blood flow. The brachial artery flow measurement shows a reduction in flow rate with a rising downstream resistance $(\mathbf{d})$ due to the closed anastomosis with the efferent vein.

Table 2. Number of patients, new VA and ECD provided per year

\begin{tabular}{|c|c|c|c|}
\hline Complications found by ECD & 2015 & 2016 & 2017 \\
\hline Number of patients in hemodialysis & 117 & 117 & 119 \\
\hline Number of patients requiring ECD for AVF dysfunction & 45 & 50 & 35 \\
\hline Hemodynamically significant stenosis & 8 & 7 & 1 \\
\hline Not hemodynamically significant stenosis & 14 & 16 & 14 \\
\hline Thrombosis & 8 & 8 & 4 \\
\hline Soft tissue hematoma (diameter $>4 \mathrm{~mm}$ ) & 36 & 24 & 1 \\
\hline Aneurysms (diameter 1.5 diameter efferent vein) & 20 & 19 & 12 \\
\hline Pseudoaneurysm & 22 & 15 & 10 \\
\hline $\begin{array}{l}\text { Echoguided cannulation (procedures not counted in the } \\
\text { total number of ECD considered per year) }\end{array}$ & 25 & 50 & 60 \\
\hline Post-cannulation complications & 25 & 10 & 2 \\
\hline Total complications found acute and chronic & 158 & 149 & 106 \\
\hline New VA provided for failure & 23 & 25 & 20 \\
\hline CVC placement for failure in AVF waiting for new AVF & 14 & 13 & 8 \\
\hline Total number of ECDs & 477 & 589 & 340 \\
\hline Complication per patient & 3.5 & 3.0 & 3.0 \\
\hline ECD per patient with dysfunction & 11 & 12 & 10 \\
\hline ECD for access cannulation on the total ECD, \% & 5 & 8 & 18 \\
\hline
\end{tabular}


evaluated and solved by following a standardized unique procedure.

In summary, the application of this protocol allows all nephrologists and vascular surgeons to study the VA in order to maintain patency and assure the best follow-up according to precise qualitative and quantitative elements. In case of acute dysfunction, the protocol allows to determine where to cannulate the access and how to perform this procedure. The standardization of the correct nomenclature for VA, and the ability of nurses to perform a quick and standardized ECD examination before, during and after the AVF cannulation contributed to the reduction of complications related to the wrong cannulation avoiding changes in the cannulation site. The early detection of acute dysfunction was identified in advance, thanks to the nurses' skill to correlate the alterations in the pressures and blood flow in the extracorporeal circulation with needle positioning and the hemodynamic status of AVF. Thus, the possibility to early identify situations at risk enabled the provision of the protocol of VA study by nephrologists in order to identify early and treat the complications.

\section{Discussion}

Optimal VA follow-up and dysfunction diagnosis require a vast set of intra- and inter-operator analyses. Standardized protocol for the study of VA may optimize these time-consuming procedures.

The utilization of a standardized tool among nephrologists and vascular surgeons makes it possible to evaluate and re-evaluate clinical situations adopting the same criteria for both the collection and the analysis of anatomic and hemodynamic alterations.

The detection of both the "early failure to mature" in the new access and the evolution in failure for the VA affected by chronic or acute dysfunction was possible, thanks to the routinely performed blood flow measurement. In such way, the comparison of the examinations carried out by nephrologists and vascular surgeons [14] was easily done due to the procedures reproducibility. The blood flow follow-up is also important for the early detection of VA dysfunction that is often diagnosed by the extracorporeal circulation alteration or by the worsening in the efficacy of extracorporeal treatment. The Doppler spectrum analysis provides information about the resistance at the VA level and can be routinely used in the follow-up. A sudden fall in the blood flow could be avoided previously by identifying those conditions in which an intervention is required.

As known, the Doppler pattern in a functional access is typical for low resistance described by a bifasic wave with a well-represented diastolic flow. Thus, changes in this pattern, compared to the previous analysis, or the presence of not typical pattern with thriphasic waves can relieve the presence of a dysfunction not still identified by the extracorporeal circulation.

B-mode and color Doppler study allows in the identification of stenosis, but only the integration of anatomical and hemodynamic parameters can provide valid information on the real operational access function, the required follow-up or therapeutic procedure (e.g., percutaneous transluminal angioplasty) [16]. It is not common to identify stenosis with a maintained blood flow; in these cases, the follow-up of the maximal systolic velocity at the stenosis in relation with the blood flow can describe the evolution of stenosis till the moment where the flow is reduced and requires an intervention. In the follow-up of VA, we can assist some situation where an anatomical stenosis is present without a fall in the blood flow, or better, there is hemodynamic compensation with a high systolic peak velocity. In these cases, as described above, the interventional approach has to be addressed in terms of the cooperation between nephrologists and vascular surgeons in order to treat only the critical hemodynamic situation with high risk to end up in a failure. In fact, in the presence of stenosis with a peak systolic velocity less than $300 \mathrm{~cm} / \mathrm{s}$ at maintained flow, the vascular assess should be observed until a situation of objective dysfunction occurs with a real drop in the flow rate. The ability to compare the peak systolic velocity over time, in the presence of an evolving stenosis, is a useful tool for assessing the development of stenosis and its hemodynamic evolution.

The monitoring of aneurysms by size, thrombosis and flow plays an essential role in AVF follow-up, but the correct measurement of descriptive parameters must always be done easily and in a reproducible way in order to allow an intra- and inter-operator reproducibility.

The use of a protocol can integrate the work of nephrologists and vascular surgeons in the diagnosis and follow-up of VA dysfunction, providing a tool capable of standardizing the language. Clinicians may benefit from a unique way to identify, analyze and describe VA alterations. Both standardized clinical and surgical approach improves patients' outcome. 


\section{Conclusions}

Patients' management in hemodialysis cannot ignore the proper monitoring of VA. In clinical practice, the most accessible, relatively inexpensive, rapid, non-invasive and repeatable method of VA monitoring is represented by the ECD. However, it is necessary to standardize it in order to be effective and represent a valid tool among the various specialists involved in the hemodialysis patient care. The introduction of a well-defined protocol based on anatomic and hemodynamic parameters could facilitate the multidisciplinary management of VA, its monitoring and the timely prevention of any complications.

The application of this method of VA analysis can be used by nephrologists and vascular surgeons in order to provide an appropriate follow-up of the chronic complication and the early and effective detection of the acute dysfunction with their rapid and effective treatment. The possibility to standardize the blood flow, the dimensions of the anatomical alteration and the complex analysis of the stenosis with its impact on the global hemodynamics of AVF allow the possibility to have a tool able to share information and competences between nephrologists, which has to use the VA for the extracorporeal circulation, and the vascular surgeons who has to intervene in the alterations only if the global access function is affected or there is the possibility of experiencing failure.

The possibility to train all nurses with a standard protocol for the ECD use in hemodialysis provides an effective, quick and helpful tool that can be routinely used before, during and after the cannulation of difficult VA. Furthermore, the ability of nurses to identify an alteration in the extracorporeal circulation due to the needle positions or AVF hemodynamics can be improved by the systematic use of ECD in order to find needle malposition, thrombosis and fall in the blood flow due to critical stenosis in evolution. This first step will be followed by the peculiar analysis of the nephrologist and the vascular surgeon who will be able to propose a surgical solution or a change in the cannulation procedures.

In our experience, theECDintroduction in the Hemodialysis Unit improved the cooperation, communication, competenceand theoutcomeofourAVF.

\section{References}

1 Lambie SH, Taal MW, Fluck RJ, McIntyre $\mathrm{CW}$ : Analysis of factors associated with variability in haemodialysis adequacy. Nephrol Dial Transplant 2004;19:406-412.

2 Moura A, Madureira J, Alija P, Fernandes JC, Oliveira JG, Lopez M, Filgueiras M, Amado L, Sameiro-Faria M, Miranda V, Vieira M, Santos-Silva A, Costa E: Type of vascular access and location in online hemodiafiltration and its association with patient's perception of health-related quality of life. J Vasc Access 2014; 15:175-182.

3 Thomas M, Nesbitt C, Ghouri M, Hansrani $\mathrm{M}$ : Maintenance of hemodialysis vascular access and prevention of access dysfunction: a review. Ann Vasc Surg 2017;43:318-327.

4 Schild AF: Maintaining vascular access: the management of hemodialysis arteriovenous grafts. J Vasc Access 2010;11:92-99.

5 Gadallah MF, Paulson WD, Vickers B, Work $\mathrm{J}$ : Accuracy of Doppler ultrasound in diagnosing anatomic stenosis of hemodialysis arteriovenous access as compared with fistulography. Am J Kidney Dis 1998;32:273-277.

6 Depairon M, Ferrier CP, Tutta P, Descombes E, van Melle G, Wauters JP: Is there a place for duplex screening of brachial artery in haemo- dialysis patients with vascular access? Vasa 2001;30:53-58.

7 Visciano B, Riccio E, De Falco V, Musumeci A, Capuano I, Memoli A, Di Nuzzi A, Pisani A: Complications of native arteriovenous fistula: the role of color Doppler ultrasonography. Ther Apher Dial 2014;18:155-161.

8 Zwiebel WJ: Duplex sonography of the cerebral arteries: efficacy, limitations, and indications. AJR Am J Roentgenol 1992;158:29-36.

9 Napoli M, Tazza L, Postorino M, Bonucchi D, Lodi M, Granata A, Lomonte C, Meola M, Spina M: [The Italian registry of vascular access]. G Ital Nefrol 2013;30:pii:gin/30.6.15.

10 Lipari G, Tessitore N, Poli A, Bedogna V, Impedovo A, Lupo A, Baggio E: Outcomes of surgical revision of stenosed and thrombosed forearm arteriovenous fistulae for haemodialysis. Nephrol Dial Transplant 2007;22: 2605-2612.

11 Cho S: The feasibility of surgical salvage of thrombosed arteriovenous fistula by an interventional nephrologist. Kidney Res Clin Pract 2017:36:175-181.

12 Jemcov TK: Morphologic and functional vessels characteristics assessed by ultrasonography for prediction of radiocephalic fistula maturation. J Vasc Access 2013;14:356-363.

13 Finlay DE, Longley DG, Foshager MC, Letourneau JG: Duplex and color Doppler sonography of hemodialysis arteriovenous fistulas and grafts. Radiographics 1993;13:983989 .

14 Grosser S, Kreymann G, Kuhns A, Greten H: [Color-coded duplex sonography in primary diagnosis, monitoring and prognostic evaluation of fibrinolytic and conservative therapy of thrombophlebitis]. Vasa Suppl 1991;32: 187-190.

15 Fumagalli G, De Pietro S, Migliori M, Ferrandello FP, Trovato F, Donadio C, Panichi V: Outcomes of vascular access care and surgery managed by interventional nephrologists: a twelve-year experience. Blood Purif 2016;42: 111-120.

16 Matsui S, Nakai K, Taniguchi T, Nagai T, Yokomatsu T, Kono Y, Mizoguchi T, Miki S, Yoshida A, Nagao K, Tsuji H, Ono S: Systematic evaluation of vascular access by color-Doppler ultrasound decreased the incidence of emergent vascular access intervention therapy and X-ray exposure time: a single-center observational study. Ther Apher Dial 2012; 16:169-172. 